\title{
SYSTEM FOR AUTOMATIC DETECTION AND ANALYSIS OF TARGETS IN FMICW RADAR SIGNAL
}

\author{
Luboš Rejfek - Zbyšek Mošna - \\ Jaroslav Urbář — Petra Koucká Knížová *
}

\begin{abstract}
This paper presents the automatic system for the processing of the signals from the frequency modulated interrupted continuous wave (FMICW) radar and describes methods for the primary signal processing. Further, we present methods for the detection of the targets in strong noise. These methods are tested both on the real and simulated signals. The real signals were measured using the developed at the IAP CAS experimental prototype of FMICW radar with operational frequency 35.4 GHz. The measurement campaign took place at the TU Delft, the Netherlands. The obtained results were used for development of the system for the automatic detection and analysis of the targets measured by the FMICW radar.
\end{abstract}

K e y w ords: power spectral density (PSD), FMICW radar, Doppler measurement, thresholding, false alert detection

\section{INTRODUCTION}

Radars are important devices usable for many types of the applications (ship or plane navigation, detection of people under an avalanche, vehicle speed measurement, area protection and other). In the Institute of Atmospheric Physics, Czech Academy of Sciences (IAP) we are developing radar for the meteorological targets detection and identification. The radar is based on two types of the radars (pulse radar and FMCW radar). Manual (human) control is necessary for the radar functionality. The operators are needed to do detection and interpretation of the targets. In this paper, we describe system for the automatic detection of the targets. Automatic detection and interpretation of targets in the radar signals has been always important. These automatic systems serve to decrease time of the operator. Another benefit is faster data processing and target interpretation compared to human operator. The signal processing must be very often realized in real-time with high precision. Principles described in present paper will be used for further advanced automatic or manual data processing development. First part of the paper describes used system. In our case, FMICW radar is used to monitor distances of the targets and Doppler shifted frequencies of the targets. Second part is about primary data processing (calculation of the spectrum, filtration and other). Third part deals with the method for the detection of targets and their separation from the spectrum and their statistical description (skewness, kurtosis). Thresholding, PSD and other methods must be used for the signal processing. Last part of this paper describes testing of data processing for different combination of used methods.

\section{FMICW RADAR}

Conceptual scheme of the FMICW radar is following: the transmitter is composed of the sweeping generator, mixer, PIN diode and generator of the carrier wave. Output from this part is connected to the circulator. The second part is the receiver, which is composed of the PIN diode, mixer and analog to digital converter (ADC). For the signal transmission and receiving one antenna setting described in [1], or antenna array described in [2] can be used. Universal scheme is in Fig. 1 and more detailed scheme is described for example in [3].

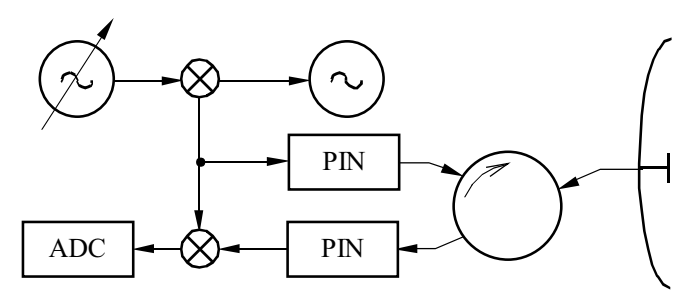

Fig. 1. Block scheme of the FMICW radar

Figure 2 provides functional scheme of the FMICW radar. Signal generated by this radar is frequency swept. Repetition time of the frequency sweeping $\left(T-T_{\text {rep }}\right)$ is set according to mode used for the measurement. rep consists of the time of the transmitting duration $\left(T_{\text {tran }}\right)$, time of the receiving duration $\left(T_{\text {rec }}\right)$ and the duration of the blind zone. The total time is denoted " $T$ ". Lost frequencies are the signals reflected from the target which return before start of signal recording. Received frequencies are used for the calculation of the target distance.

Signal received from measurement at the TU Delft is shown in the Fig. 3. Whole duration of one realization re-

\footnotetext{
* Department of Aeronomy, Institute of Atmospheric Physics CAS, Prague 4, Czech Republic, lre@ufa.cas.cz, jur@ufa.cas.cz, pkn@ufa.cas.cz, zbn@ufa.cas.cz,
} 


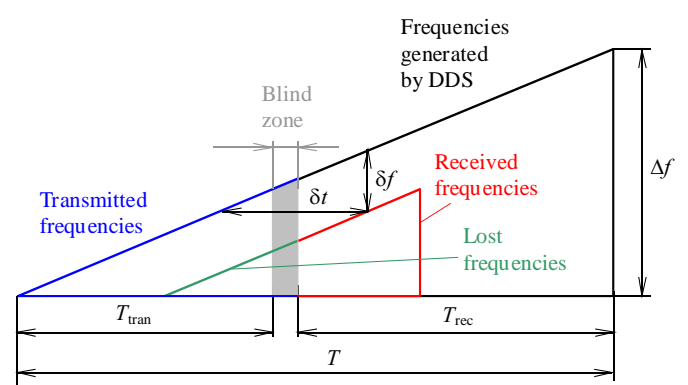

Fig. 2. Functional scheme of the FMICW radar. [4]

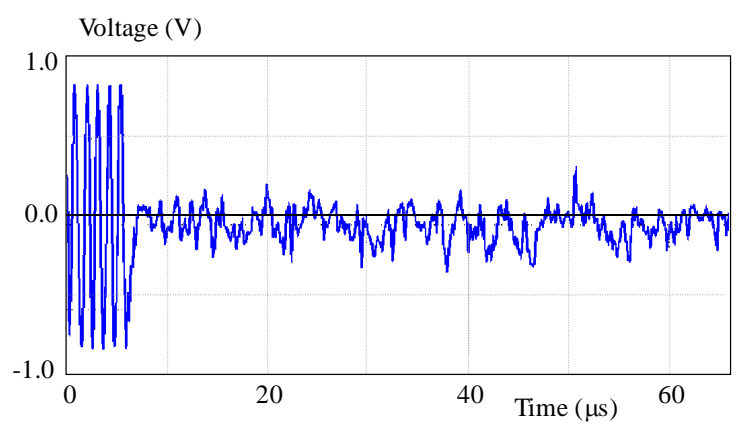

Fig. 3. An example of the received signal with a chimney as a target

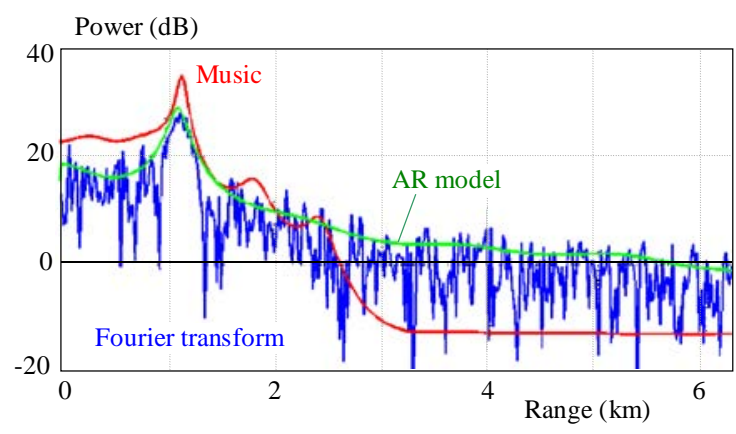

Fig. 4. Comparison of the PSD methods

ceiving/record is $660 \mu \mathrm{s}$. The part of interest (containing information about the target) lasts approximately $7.2 \mu \mathrm{s}$. Measured data are saved as a square matrix with 660 rows for realizations and 660 lines for repetition of measurements. Another source of these signals can be a simulator. An example of FMICW radar simulator is described in $[4]$.

\section{METHODS FOR THE PROCESSING OF THE SIGNAL}

Radar signal must be preprocessed before detection of the targets. The first step, performed both for the FMCW, or FMICW radar, is transformation of the signal from the time domain to the frequency domain (range). The second step is transformation of the Doppler decomposition. As the third step, filtration can be used.

\subsection{Power Spectral Density (PSD) Methods}

PSD methods are divided into two groups. The first group represents nonparametric methods (periodograms) and the second group consists of parametric methods (autoregressive model (AR), multi signal classification (MUSIC), Eigen vector algorithm, minimum norm. algorithm and others). Other applicable methods are described for instance in [5-7].

Periodograms can be calculated directly using Fourier transform from the data sequence, or indirectly using Fourier transform from autocovariance sequence of the data. Direct version, see [6], is calculated according to

$$
P_{x x}\left(e^{j 2 \pi f_{k}}\right)=\frac{1}{N}\left|\sum_{n=0}^{N-1} x[n] e^{-j k(2 \pi / N) n}\right|^{2},
$$

where $P$ is power of the spectral element, $N$ is length of the data sequence, $x[n]$ is $n^{\text {th }}$ element of the data sequence and $\mathrm{k}$ reaches values from 0 to $N-1$.

AR model is based on the infinite impulse response filter. The spectrum is calculated according to (2). YuleWalker equations can be used for the calculation as described in [8]. Another possibility is the use of finite impulse response filter (MA model) or their combination (ARMA model).

$$
P_{x x}\left(e^{j 2 \pi f}\right)=E\left(|\omega[n]|^{2}\right)\left|\left(1+\sum_{i=1}^{m} a_{i} e^{j 2 \pi f}\right)^{-1}\right|^{2}
$$

where $a$ are poles of transfer function of the system, $m$ is the number of the poles and $\omega$ is white noise.

MUSIC method is based on the decomposition of the autocovariance matrix to the eigenvalues and eigenvectors. This method can be performed for the signal subspace, or for the noise subspace. Equation (3) is MUSIC method for the signal subspace. For more details see [7].

$$
P_{x x}\left(e^{j 2 \pi f}\right)=\frac{1}{\sum_{i=0}^{p-1}\left|e^{H} v_{i}\right|^{2}},
$$

where $v_{i}$ is eigenvector, $e^{H}$ is vector of the exponential functions and $p$ is the used parameter.

Comparison of the Fourier transform, MUSIC method and AR model is shown in the Fig. 4. One can see that spectrum and pseudo spectra are in agreement. Radar signals must be processed in real time. For the following processing Fast Fourier Transform (FFT) algorithm is used. Comparison of the time demands of the parametric methods is described in [8]. Only a small part of the recorded signal represents the useful signal in FMICW radars. Correction of the received PSDs must be used for the correct interpretation. See for instance [9]. 


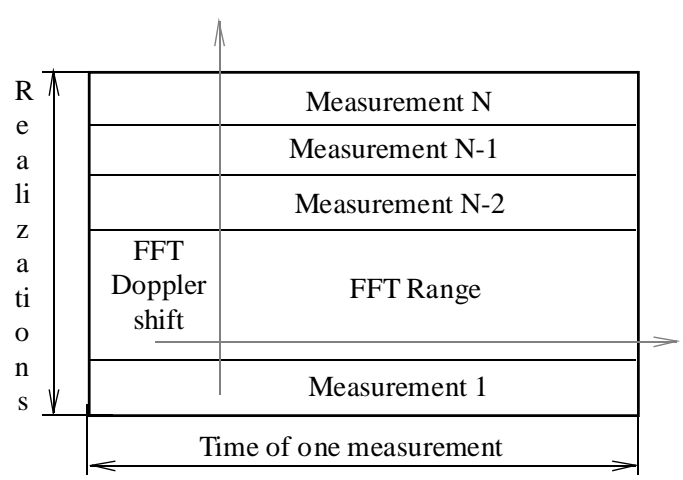

Fig. 5. Implementation of the PSD method for the Doppler measurement in the FMCW or FMICW radars. [3]

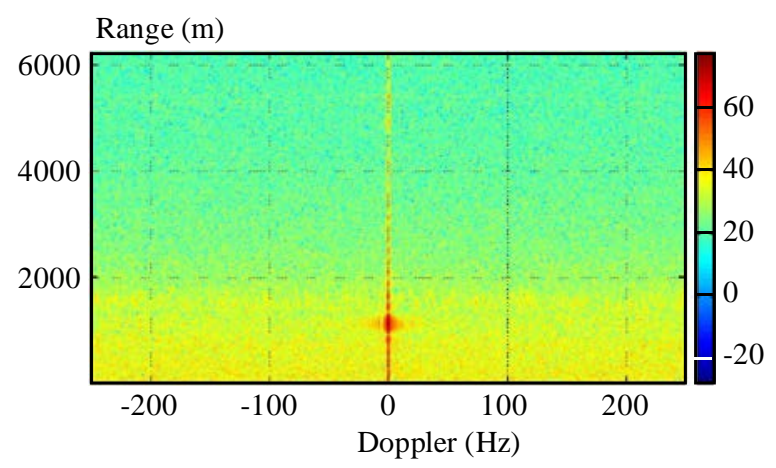

Fig. 6. Signals transformed from the time domain to the $2 \mathrm{D}$ frequency domain. The first frequency dimension is normalized to ranges and the second frequency dimension is normalized to Doppler shifts

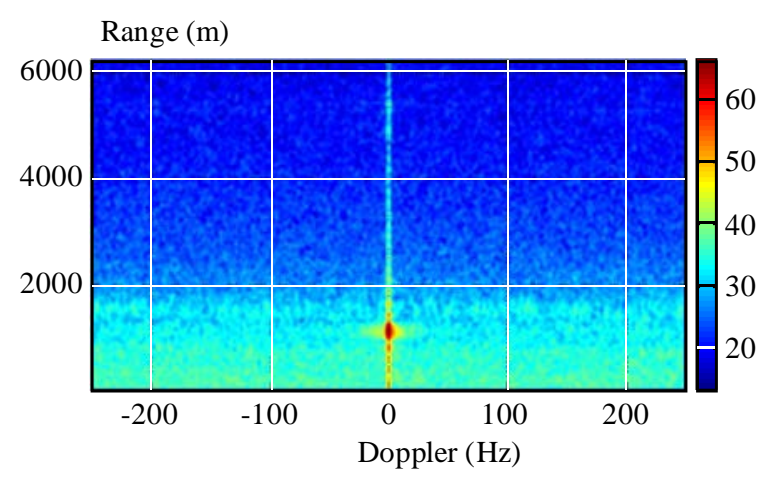

Fig. 7. Transformed signals (Figure 6.) after 2D filtration

\subsection{Measurement of the Doppler Frequencies}

Doppler frequencies can be measured by two methods. First type is based on using generator without frequency sweeping. This type is used only for the measurement of the Doppler frequencies, but the ranges cannot be determined. Second type is algorithm for measurement of the Doppler frequencies for the separated ranges. The first step of the algorithm represents Fourier transform of measurements in time domain. The second step is Fourier transform through the ranges in complex spectrum. The third step comprises conversion from the non-centered spectrum to the centered spectrum according to both diagonals. This algorithm is shown in Fig. 5.
Figure 6 shows an example of the signals processed according to above described algorithm of Doppler frequency measurement. The experimental target was a chimney measured at TU Delft. It was static target in the distance $1139 \mathrm{~m}$.

\subsection{Filtration of the Transformed Signal}

For the elimination of the extreme points occurring due to a noise a filtration should be applied. Moving average filter is a simple filter for the removal of the high frequency events. $2 \mathrm{D}$ data can be filtered by application of horizontal line filter, vertical line filter, diagonal line filter, or by the matrix filter. Figures 6 and 7 show the same signal before and after filtering by the square matrix $(4 \times 4)$. All gains are equal to 1 . Equation (4) represents filtered power element by the weighted moving average.

$$
P_{x, y}=\frac{1}{A} \sum_{i=-\frac{N}{2}}^{\frac{N}{2}} \sum_{i=-\frac{M}{2}}^{\frac{M}{2}} P_{x+i, y+j} a_{i, j}
$$

where $N$ is the length of the vertical vector, $M$ is the length of the horizontal vector, $P_{x+i, y+j}$ is the original power, $a_{i, j}$ is the gain of the element and $A$ is the sum of gains of these elements.

\section{METHODS FOR THE DETECTION AND ANALYSIS OF THE TARGETS}

The first step of the analysis is detection of the targets from the preprocessed signals. Then detected targets can be analyzed in detail. Doppler range, distance and length of the target are derived parameters by the FMICW radar. Thresholding and false alert detection can be further applied. It is crucial to determine the target position, length and Doppler range. Another possibility is, for example, the estimation of the kurtosis and skewness.

\subsection{Thresholding of the Transformed Signal}

Thresholding is used for the separation of the targets. The separation level (threshold) is constant and exceeding PSDs are attributed to the targets and the smaller PSDs to the noise as shown in the condition (5). Target after thresholding is shown in Fig. 8. Both plots show the same target without (left) and with (right) signal filtration. In this example the threshold is fixed at the $10 \%$ of the maximum power.

$$
P_{x, y} \geq P_{\min },
$$

where $P_{x, y}$ is original power and $P_{\min }$ is minimal power.

\subsection{False Alert Detection}

Sometimes, false targets can be found after thresholding. One of the methods for removal of the false targets is a videodetection. This is important in case of weak targets. Figure 9 shows strong target with very low threshold value (for the simulation of the weak target). The left part 


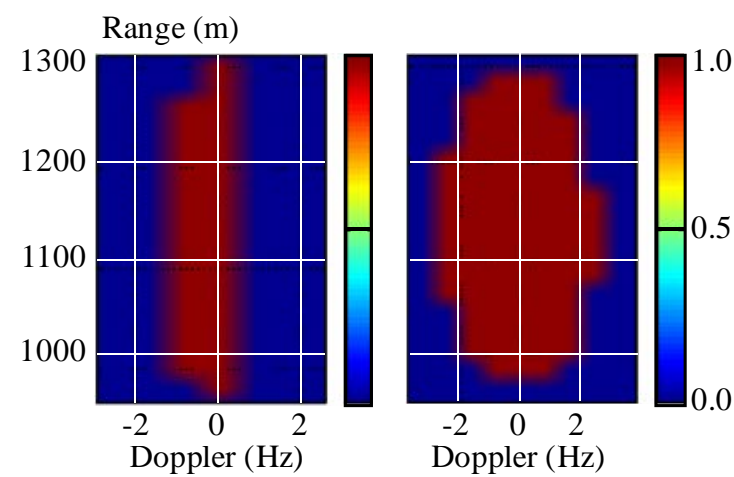

Fig. 8. Target after thresholding according to $10 \%$ level of the signal. Left part is for the thresholding of the original ranges of the Doppler values and right part is for the thresholding of the filtered ranges of the Doppler values

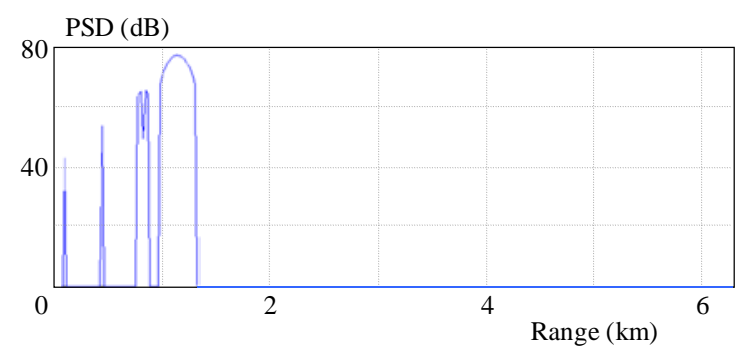

Fig. 10. Range profile estimated by the use of the PSD

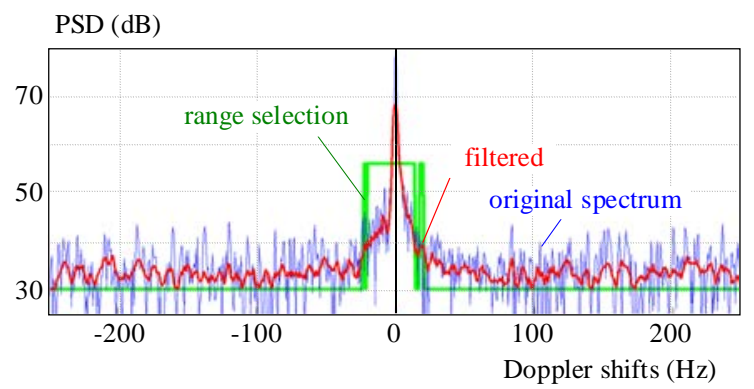

Fig. 12. Profile of the Dopplers for the target (chimney) distance

is for the ranges and Doppler spectra with noise and the right part is for the result of videodetection. Matrix with 1 row and 10 columns was used for the videodetection.

Principle of the videodetection is similar to the filtration. The difference is in setting all the gains equal to 1 and input powers from the $2 \mathrm{D}$ spectrum after thresholding. If output signal according to modified equation (4) is higher than the set value, target is confirmed, otherwise the target is rejected.

\subsection{Detection of the Range}

Detections of the target ranges can be performed by mean of range profile. This distance profile can be made by the use of the power peaks in the spectrum. This profile example is shown in Fig. 10. The second possibility is application of the range profile in accordance with the number of positive detections in Doppler range (NPDDR). Example of such profile is shown in Fig. 11. Distance of

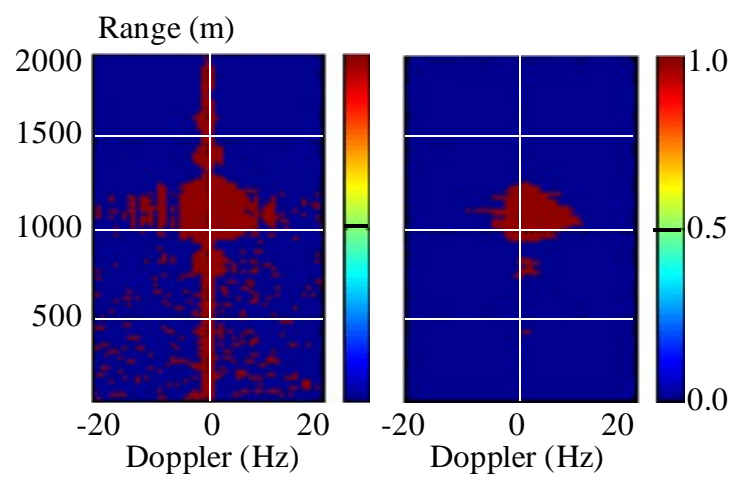

Fig. 9. Weak target in the noise before use of the videodetection and after use of the videodetection. Threshold value is $0.03 \%$ length of the sequence is 10 samples and condition of acceptance is $75 \%$ of positive detections

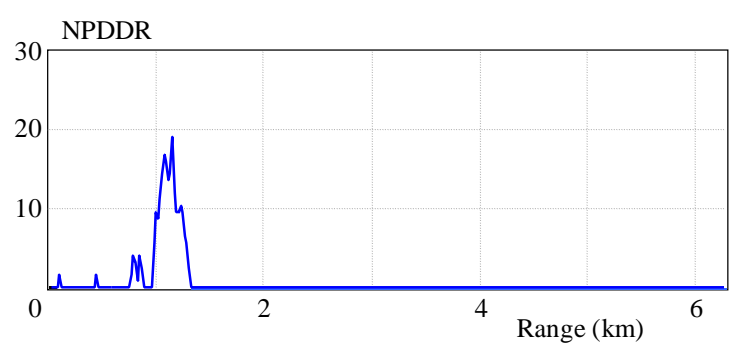

Fig. 11. Range profile estimated by the use of the positive Dopplers

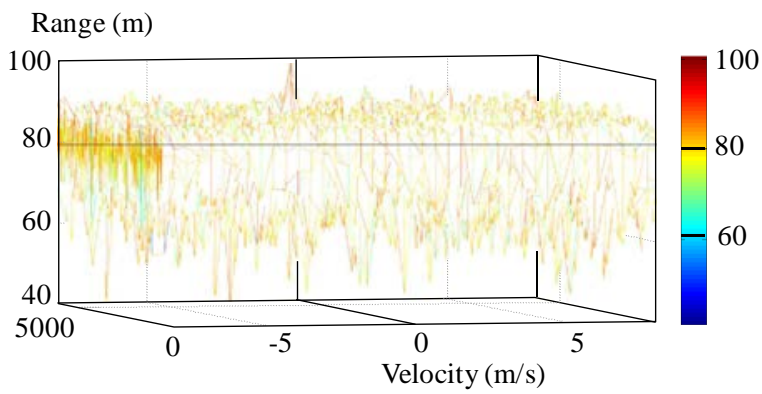

Fig. 13. Example of the simulated signals $2 \mathrm{D}$ spectrum (ranges, velocity). velocity of the target is $2 \mathrm{~m} / \mathrm{s}$ and range of the target is $3 \mathrm{~km}$

the targets is derived using the algorithms of the local maximum detection. One example of the algorithm for local maximum detection is described in [10]. Figures 10 and 11 show false alerts on ranges smaller than $1 \mathrm{~km}$, which are caused by the length of the reflected signal in the recorded sequence. Power spectrum of this signal is defined as (6). Secondary peaks are not automatically removed by the signal processing in this case.

$$
P(x)=\left(\frac{\sin x}{x}\right)^{2}
$$

\subsection{Doppler Range, Skewness and Kurtosis of the Dopplers}

Doppler range is defined by use of the thresholding. Figure 12 shows example of the Doppler profile of the static target (chimney) on the distance. Small shifts and shape deformation in spectrum can be caused for example 


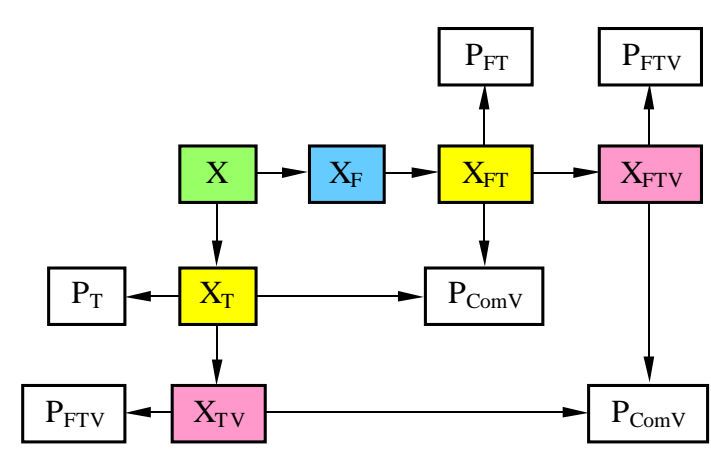

Fig. 14. Scheme of the automatic detection algorithms

Table 1. Success of the automatic processing by the use of the PSD profile

\begin{tabular}{llllll}
\hline PSD & \multicolumn{2}{l}{ SNR $=3 \mathrm{~dB}$} & NOISE & \multicolumn{2}{l}{ Strong Target } \\
Interval & Distance & Velocity & Distance & Distance & Velocity \\
\hline $\mathrm{P}_{\mathrm{T}}{ }^{\mathrm{a}}$ & $0 \%$ & $0 \%$ & $0 \%$ & $0 \%$ & $0 \%$ \\
$\mathrm{P}_{\mathrm{TV}}{ }^{\mathrm{b}}$ & $71 \%$ & $70 \%$ & $77 \%$ & $6 \%$ & $6 \%$ \\
$\mathrm{P}_{\mathrm{Com}}{ }^{\mathrm{c}}$ & $73 \%$ & $72 \%$ & $67 \%$ & $47 \%$ & $47 \%$ \\
$\mathrm{P}_{\mathrm{FT}}{ }^{\mathrm{d}}$ & $0 \%$ & $0 \%$ & $0 \%$ & $37 \%$ & $37 \%$ \\
$\mathrm{P}_{\mathrm{FTV}}{ }^{\mathrm{e}}$ & $1 \%$ & $1 \%$ & $13 \%$ & $74 \%$ & $74 \%$ \\
$\mathrm{P}_{\mathrm{ComV}}$ & $92 \%$ & $91 \%$ & $100 \%$ & $74 \%$ & $74 \%$ \\
\hline
\end{tabular}

Table 2. Success of the automatic processing by the use of the NPDDR profile

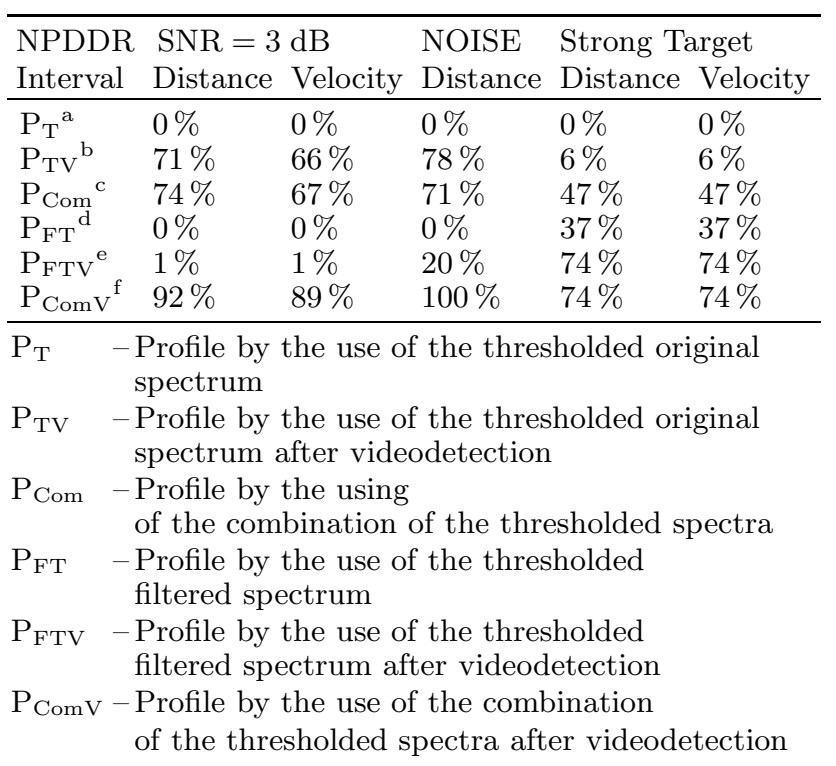

by the wind influence on the chimney. The original spectrum (blue line), the filtered spectrum (red line) and the selection of the Doppler range of the target(green line) are shown.

PSD, where dominant target/peak can be found correspond to airplane, ship, buildings etc. PSD without dominant signal represent inhomogeneous target (eg, flock of birds and other).

Dominant target can be found by use of the kurtosis. Deflection of the target in the range can be detected by the using of skewness. It is important for the inhomogeneous targets.

\section{EXPERIMENT AND VERIFICATION}

Tests of the process variations were performed on the simulated signals. Signals were generated by the modified simulator described in [3]. One example of the 2D spectrum of the simulated signal is shown in Fig. 13. Doppler frequencies are in this case converted into the corresponding velocities. $2 \mathrm{D}$ spectra of the simulated signals serve as input for the data process. Spectra are calculated from the 42 time-signals. Experiment is realized on the 100 spectra for the SNR $=3 \mathrm{~dB}, 100$ spectra for the noise without signal and 100 spectra for the strongly reflecting target.

Experimental test of the algorithms for the automatic spectrum processing is shown in Fig. 14. In the scheme block "X" (light green) represents original spectrum, block " $\mathrm{X}_{\mathrm{F}}$ " (blue) stands for the filtered spectrum (used is $2 \mathrm{D}$ filter of the 2nd order), blocks " $\mathrm{X}_{\mathrm{T}}$ ", " $\mathrm{X}_{\mathrm{FT}}$ " (yellow) indicate the thresholded spectrum (threshold values are in this case calculated as median value of the maximum values for the distance vectors), blocks " $\mathrm{X}_{\mathrm{TV}}$ ", "X $\mathrm{X}_{\mathrm{FTV}}$ " (magenta) are the thresholded spectrum after videodetection (size of the window for the videodetection is 3 points) and rest (white) blocks denote the estimated profiles of the distances. The scheme is similar for both PSD and NPDDR profile experiments. Results of the profile based on PSD are in Tab. 1 and results for the profile from the NPDDR are described in Tab. 2.

The tests show that the best results are achieved using the $\mathrm{P}_{\mathrm{ComV}}$ profile. Merging of the very nearby targets was used for removing of the noise. This technique caused that similar results of distance and velocity were obtained in case of the strong targets. Use of both profiles leads to similar results. Significant part of the errors created by the strong targets analyses was caused by the secondary peaks in the spectra. These peaks could be detected and removed (their function is described in (6)) for better results of the spectrum analyses.

\section{CONCLUSION}

Comparison of the algorithms for the FMICW radar signals processing was described in this paper. From the results it is evident that the most convenient method is comparison of the both thresholded spectra after videodetection. This can be used for the automatic detection of the targets and archiving of the information about them. Data can be used for the atmospheric research (meteorological version of the radar), for the study of car velocities (transport version of the radar) and for other scientific or commercial applications. Using real data it is possible to observe the noise level is higher for near distances and decreases with the distance which means that signal noise ratio for near targets is smaller compared to distant monitored area. This behavior can be minimized by removing 
of system background as described eg in [4]. The system will be further improved in near future (implementation of advanced methods for study of characteristics described here, or implementation of the methods for the study of additional characteristics are possible).

\section{Acknowledgement}

Thanks go to the grants GAČR P209/12/2440 and (15-24688S). The operation of developed FMICW radar was tested during experimental campaign at the Delft University of Technology. Authors gratefully acknowledge the hospitality, support and useful discussions of the Microwave Sensing, Signals and Systems (MS3) group of TU Delft, especially Mr Fred van der Zwan and Dr Oleg Krasnov.

\section{REFERENCES}

[1] ZALABSKY, T.-BEZOUSEK, P.: TEM Horn Antenna for High Energy Pulse Emission, Radioelektronika 2013, 23rd International Conference, pp. 92-95,

http://ieeexplore.ieee.org/stamp/stamp.jsp?tp= \&arnumber $=6530898 \&$ isnumber $=6530881$.

[2] SHEJBAL, T.-BEZOUSEK, P.-ZALABSKY, T. : Active Antenna Array Concepts for Precision Approach Radar, ELMAR, 56th International Symposium, 10-12 Sept. 2014, http://ieeexplore.ieee.org/stamp/stamp.jsp?tp= \&arnumber $=6923355 \&$ isnumber $=6923293$.

[3] MANDLIK, M.-BRAZDA, V.: FMICW Radar Simulator, Radioelektronika, 25th International Conference, 21-22 April 2015, http://ieeexplore.ieee.org/stamp/stamp.jsp?tp= \&arnumber $=7129054 \&$ isnumber $=7128969$.

[4] REJFEK, L.-BEZOUSEK, P.-FISER, O.-BRAZDA, V.: FMICW Radar Simulator at the Frequency $35.4 \mathrm{GHz}$, Radioelektronika, 24th International Conference, 15-16 April 2014, http://ieeexplore.iee.org/stamp/stamp.jsp?tp= \&arnumber $=6828452$ \&isnumber $=6828396$.

[5] SHEJBAL, T. : Improvement Methods of Angular Resolution of Antenna Array, Microwave Techniques (COMITE), Conference, 22-23 April 2015, http://ieeexplore.ieee.org/stamp/ stamp.jsp?tp $=$ \&arnumber $=7120314 \&$ isnumber $=7120218$.

[6] SMÉKAL, Z.: Digital Signal Processing (Číslicové zpracování signálu), skriptum, Vysoké Učení Technické v Brně, Fakulta elektrotechniky a komunikačních technologií, 2009. (in Czech)
[7] STOICA, P.-MOSES, R.: Introduction to Spectral Analysis, Prentice Hall, Upper Saddle River, 1997.

[8] REJFEK, L.-BURESOVA, D.-FISER, O.-BRAZDA, V.: Comparison of Parametric Methods for Radar Signal Processing, Radioelektronika, 25th International Conference 21-22 April 2015, pp. 141-144, http://ieeexplore.ieee.org/stamp/ stamp.jsp?tp $=$ \&arnumber $=7128986 \&$ isnumber $=7128969$.

[9] REJFEK, L.-BERAN, L.-FISER, O.: Correction of Radar Received Signal, Radioelektronika, 25th International Conference, 21-22 April 2015, pp. 191-194,

http://ieeexplore.ieee.org/stamp/stamp.jsp?tp= \&arnumber $=7129006 \&$ isnumber $=7128969$.

10] SCHOLKMANN, F.-BOSS, J.-WOLF, M. : An Efficient Algorithm for Automatic Peak Detection in Noisy Periodic and Quasi-Periodic Signals, Algorithms 2012, pp. 588-603, DDECS Tallinn, Estonia, April 18-20.

Received 5 October 2015

Luboš Rejfek was born in 1985 in Chrudim, Czechoslovakia, graduated (Master degree) in 2011 at the Department of Electrical engineering of the Faculty of Electrical Engineering and Informatics at the University of Pardubice. At present he is $\mathrm{PhD}$ student at the same department and is employed at the Institute of Atmospheric Physics of the Czech Academy of Sciences.

Zbyšek Mošna was born in 1978 in Prague. He defended his $\mathrm{PhD}$ in 2014 at the Faculty of Mathematics and Physics of the Charles University in Prague. He is engaged in the field of ionospheric variability, vertical coupling and effects of solar, geomagnetic, and neutral atmosphere activity into the ionosphere. He works at the Institute of Atmospheric Physics of the Czech Academy of Sciences.

Jaroslav Urbář was born in 1984 in Kladno, Czechoslovakia, received his Master degree in Space science and engineering in 2010 from the Lulea University of Technology, department of space science in Kiruna. At present he is PhD student at the Faculty of Mathematics and Physics of the Charles University in Prague and is employed at the Institute of Atmospheric Physics of the Czech Academy of Sciences.

Petra Koucká Knížová was born in 1972 in Prague. She defended her PhD in 2002 at the Faculty of Mathematics and Physics of the Charles University in Prague. Her primary interest is ionospheric variability, especially the wave-like variations in the ionospheric plasma and vertical coupling process in the neutral atmosphere-ionosphere system. She works at the Institute of Atmospheric Physics of the Czech Academy of Sciences. 